\title{
Flocculation and percolation in reversible cluster-cluster aggregation
}

\author{
Sujin Babu, Manuel Rottereau, Taco Nicolai, Jean-Christophe Gimel, ${ }^{*}$ and Dominique Durand \\ Polymères Colloïdes Interfaces, CNRS UMR6120, \\ Université du Maine, F-72085 Le Mans cedex 9, France
}

(Dated: March 6, 2006)

\begin{abstract}
Off-lattice dynamic Monte-Carlo simulations were done of reversible cluster-cluster aggregation for spheres that form rigid bonds at contact. The equilibrium properties were found to be determined by the life time of encounters between two particles $\left(t_{e}\right)$. $t_{e}$ is a function not only of the probability to form or break a bond, but also of the elementary step size of the Brownian motion of the particles. In the flocculation regime the fractal dimension of the clusters is $d_{f}=2.0$ and the size distribution has a power law decay with exponent $\tau=1.5$. At larger values of $t_{e}$ transient gels are formed. Close to the percolation threshold the clusters have a fractal dimension $d_{f}=2.7$ and the power law exponent of the size distribution is $\tau=2.1$. The transition between flocculation and percolation occurs at a characteristic weight average aggregation number that decreases with increasing volume fraction.
\end{abstract}

PACS numbers: 05.10.Ln, 82.70.Dd, 82.70.Gg

\section{INTRODUCTION}

Random aggregation of small particles is a commonly observed phenomenon and may lead to the formation of gels [1-4]. The initial stage of the aggregation process in dilute systems has been extensively studied using computer simulations [5-9]. Kinetic equations with the appropriate kernels have been used to model the growth [8-10]. Much less attention has been given to the situation where the clusters can no longer be considered dilute [11-14]. As the clusters grow their cumulated volume fraction increases and at some point they will start to interpenetrate. Eventually irreversible aggregation leads to gelation at any concentration. If the aggregation process is reversible a steady state will be reached at a more or less advanced stage depending on the ratio of the aggregation and fractionation rate [15-24]. Also in this case the clusters may percolate the system, but now the gel is only transient [18, 19, 25-27].

In recent work we have simulated irreversible diffusion limited cluster-cluster aggregation (DLCA) from the initial state of monomers to the final state where all particles form a single percolating cluster $[28,29]$. We have shown that as long as the average distance between the clusters is large (flocculation regime) they have a selfsimilar structure characterized by a fractal dimension $d_{f}=1.8$. With increasing cluster size the average distance between the clusters decreases and at a characteristic aggregation number $\left(m=m_{c}\right)$ and radius of gyration $\left(R_{g}=R_{c}\right)$ they fill up the whole space and start to interpenetrate. The aggregation of clusters larger than $R_{c}$, which leads to gelation, can be described by the percolation model $[13,14,28,30]$. As a consequence the structure of the clusters on length scales larger than $R_{c}$ is characterized by $d_{f}=2.5$. Also the size distribution changes from relatively monodisperse for clusters formed

*Electronic address: Jean-Christophe.Gimel@univ-lemans.fr in the flocculation regime, i.e. $m<m_{c}$, to the power law size distribution for clusters formed in the percolation regime: $N(m) \propto m^{-2.2}$ for $m>m_{c}$.

This paper reports a study of the effect of bond breaking on the aggregation of spheres. A bond is formed at collision with probability $\alpha$, and the bond breaks at each diffusion step with probability $\beta$. The two limiting cases of diffusion (DLCA) and reaction limited (RLCA) cluster aggregation are obtained by setting $\alpha=1$ and $\alpha \ll 1$, respectively. If $\alpha / \beta$ is small the equilibrium state contains essentially monomers, while if it is large most particles will be part of a single percolating cluster. The fact that the particles stick only at contact implies that solely binary collisions occur and thus no loops can be formed. Since the bonds are rigid loops cannot be formed at a later stage either. As a consequence, the average number of neighbours per particle cannot exceed two. This means that reversibility does not lead to densification and phase separation. The latter does occur if the interaction range is finite or if the bonds are not rigid $[22,25,31,32]$. The present model of cluster-cluster aggregation is thus probably the simplest one that includes reversibility. We note that for lattice simulations more than one contact can be formed per collision so that densification and phase separation do occur [19, 33].

The paper is organized as follows. We will first explain the off-lattice simulation method used for this study and discuss the distinction between encounters and collisions. This distinction was discussed earlier by Odriozola et al. $[10,34]$ in the context of irreversible RLCA and reversible DLCA. During one encounter the particles may have many correlated collisions before they move apart. In order to model the early stage of irreversible RLCA or reversible aggregation it is necessary to account for the number of collisions per encounter. It will be shown that the important parameter in the simulations is not the life time of a single bond, but the life time of an encounter $\left(t_{e}\right) . t_{e}$ is a function both of $\alpha / \beta$ and the number of collisions per encounter. The latter increases when the step size of the Brownian motion $(s)$ is decreased. However, 
the simulations are independent of $s$ if $\alpha / \beta$ is chosen in such a way that $t_{e}$ is constant.

Next we will discuss the results obtained in the flocculation regime in terms of mean field theory using kinetic equations containing both an aggregation and a fragmentation kernel. These results will be compared with earlier simulations of reversible DLCA reported by Odriozola et al. [34]. Finally, we will show that if the fragmentation rate is decreased transient gels are formed and that the sol-gel transition can be described by the percolation model.

\section{SIMULATION METHOD}

A cluster is chosen randomly and is moved with step size $s$ in a random direction. The movement of a cluster occurs with a probability inversely proportional to its diameter. This means that the clusters diffuse with a diffusion coefficient that is inversely proportional to their diameter. We thus simulate Brownian motion. We have shown in Ref. [28] that this method gives the correct time dependence of the average molar mass for the case of irreversible DLCA. If the displacement of a cluster leads to overlap between two spheres or the wall it is truncated at contact. The displacement procedure is repeated a number of times equal to the total number of clusters in the box. Then bonds are formed with probability $\alpha$ between spheres in contact and bonds are broken with probability $\beta$. In the present simulation the attempt rate to break and form bonds is the same as the attempt rate for each cluster to move one step. Notice that large clusters need on average many attempts before they move. We have tested the effect of varying the attempt rate to break and form bonds. No significant effect is observed if the rate is increased, but, of course, it increases the CPU time. If the attempt rate to break and form bonds is slower that of the movement an effect is observed that is similar to that observed if both $\alpha$ and $\beta$ are reduced by the same factor, see below and Fig. 3 .

This cluster formation procedure defines the clusters that are moved in the subsequent displacement procedure. After these two procedures the simulation time $\left(t_{\text {sim }}\right)$ is incremented by 1 and the whole process is repeated until equilibrium is reached. Contrary to Odriozola et al [34]. we do not move the clusters automatically apart when the bond is broken. If the clusters have not moved apart in the following movement procedure the bonds will be formed again with probability $\alpha$ in the next cluster formation procedure.

The starting configuration consists of $N$ randomly positioned spheres with unit diameter in a box with size $L$ at volume fraction $\phi$. The physical time $(t)$ unit is defined as the time needed for an individual sphere to diffuse a distance equal to its diameter: $t=s^{2} \cdot t_{\text {sim }}$. For example the time unit would be $0.4 s$ for spheres with a diameter of $1 \mu \mathrm{m}$ in water at $20^{\circ} \mathrm{C}$. The displacement of the clusters is diffusional and independent of $s$ for dis- tances larger than $3 s$. As mentioned above, bonds are attempted to be broken and formed at each simulation step. As a consequence the attempt rate in terms of the physical time decreases with increasing step size and is equal to $s^{-2}$.

The effect of finite box size was studied and is negligible as long as the largest cluster in the box is smaller than $L$. The results shown in this paper are not influenced by finite size effects.

\section{ENCOUNTER VERSUS COLLISION}

Two initially decorrelated spheres that collide will have on average $N_{c o l}$ collisions before they move apart and their positions become decorrelated again. The number of collisions per encounter is, of course, independent of $\alpha$ and $\beta$, but does depend on $s$. We have done simulations following the diffusion of two spheres that collide at $t=0$. They show that $N_{\text {col }}$ increases linearly with $s^{-1}: N_{c o l}=2.36 / s+1$, see Fig.1. Here, we also count as a collision the event when two unbound spheres at contact move into each others direction and therefore remain at contact after the displacement procedure. This event will account on average for halve of the collisions. Each collision leads to a delay of the diffusion process proportional to $\alpha / \beta$ corresponding to the average time during which the particles are bound per collision. We will call the average time that the particles are bound during an encounter the life time of an encounter, which is a linear function of $\alpha / \beta$ and $s$, see appendix 1:

$$
t_{e}=(2.36+s) \cdot \alpha / \beta \cdot s
$$

The distinction between collisions and encounters is important, because the properties of the system are not determined by the life time of a single bond, but by the total time that two spheres are bound during an encounter. We have verified that the same simulation results are obtained for different values of $s$ and $\alpha / \beta$ if $t_{e}$ remains the same. However, $s$ needs in any case to be much smaller than the average distance between the surfaces of the spheres in order to assure Brownian motion between encounters as was discussed elsewhere for the case of irreversible aggregation [35].

In RLCA, encounters between larger clusters occur, which may have a larger number of correlated collisions before they move apart. In this case the bonds are permanent and the clusters retain their integrity during diffusion. In reversible aggregation, however, the clusters are transient and it is in our opinion more useful to consider encounters between larger clusters as a collection of a number of individual sticky spheres. At any time an encounter may involve many clusters, contrary to the assumption of Odriozola et al. [34] who just considered encounters between only two stable clusters. At each simulation step any of the bonds in the cluster has probability $\beta$ to break. As a consequence, the average life 


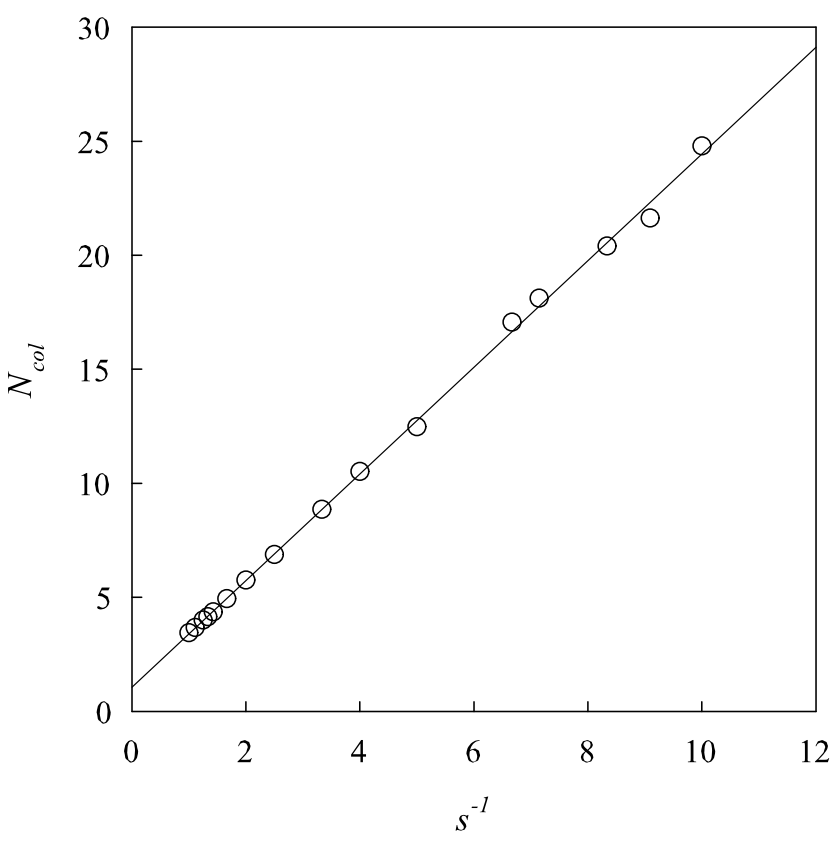

FIG. 1: Dependence of the number of correlated collisions on the step size. The straight line represents a linear least square fit : $N_{c o l}=1+2.36 / s$.

time $\left(t_{l}\right)$ of a cluster with aggregation number $m$ before any of its bonds breaks, decreases rapidly with increasing $m$ and is proportional to $s^{2}$, see appendix 2 . Large clusters can only diffuse a mean square distance of $\left.<r^{2}\right\rangle=s^{2} /\left(\beta \cdot m^{1+1 / d_{f}}\right)$, before one of the bonds breaks. For a given $t_{e}, \beta \propto s$ (see Eq.1) so that $<r^{2}>\propto s$. It follows that when a cluster fragments each of the parts will itself fragment before the parts have diffused apart by a significant distance is $s$ is small.

\section{FLOCCULATION}

The number averaged $\left(m_{n}\right)$ and the weight averaged $\left(m_{w}\right)$ aggregation number of the clusters are defined as:

$$
\begin{aligned}
& m_{n}=\frac{\sum m \cdot N(m)}{\sum N(m)} \\
& m_{w}=\frac{\sum m^{2} \cdot N(m)}{\sum m \cdot N(m)}
\end{aligned}
$$

Fig. 2 shows the time dependence of $m_{n}$ and $m_{w}$ for different values of $t_{e}$ at $\phi=0.5 \%$ using $\alpha=1$, i.e. reversible DLCA. The initial growth of $m_{n}$ and $m_{w}$ follows that of irreversible DLCA $\left(t_{e}=\infty\right)$ as long as relatively few bonds are broken. At later times the growth of $m_{n}$ slows down rapidly, while $m_{w}$ continues to increase,
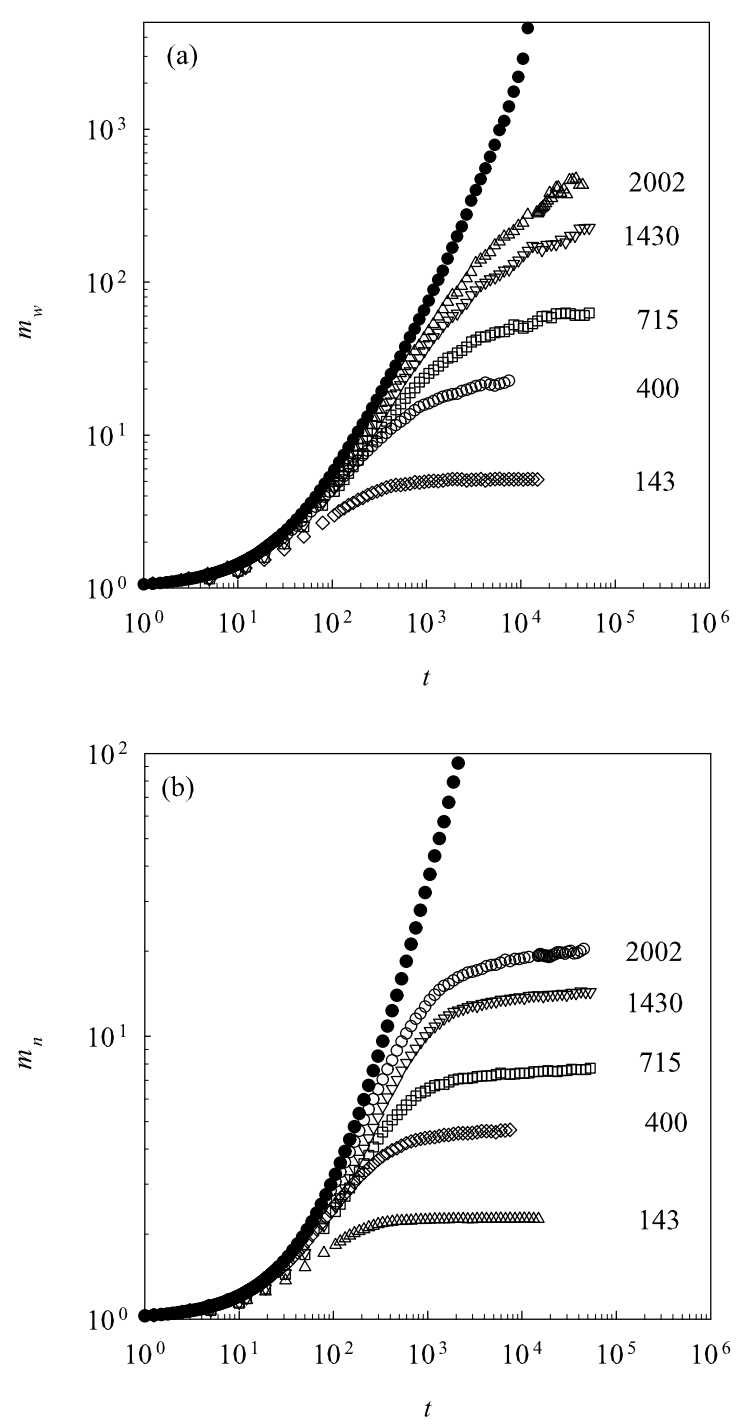

FIG. 2: Time dependence of the weight averaged (a) and the number averaged (b) aggregation number for $\phi=0.5 \%$ at different values of $t_{e}$ indicated in the figure. The closed symbols represent the result of irreversible DLCA.

which means that the polydispersity increases. Finally also the growth of $m_{w}$ slows down until equilibrium values are obtained for $t \gg t_{e}$. Similar behaviour is seen at all volume fractions.

The effect of varying $\alpha$ for the same $t_{e}$ is shown in Fig.3. Decreasing $\alpha$ slows the aggregation down, but does not modify the equilibrium structure. We have verified for different values of $\phi$ and $t_{e}$ that the equilibrium structure is independent of $\alpha$ at fixed $t_{e}$. There is thus no distinction between reversible DLCA and RLCA at equilibrium. Therefore we have generally used $\alpha=1$ for which equilibrium is reached quickest.

The size distribution and the structure of the clusters were determined at equilibrium for a range of $t_{e}$. Fig.4 shows that $m$ has a power law dependence on $R_{g}$, which 


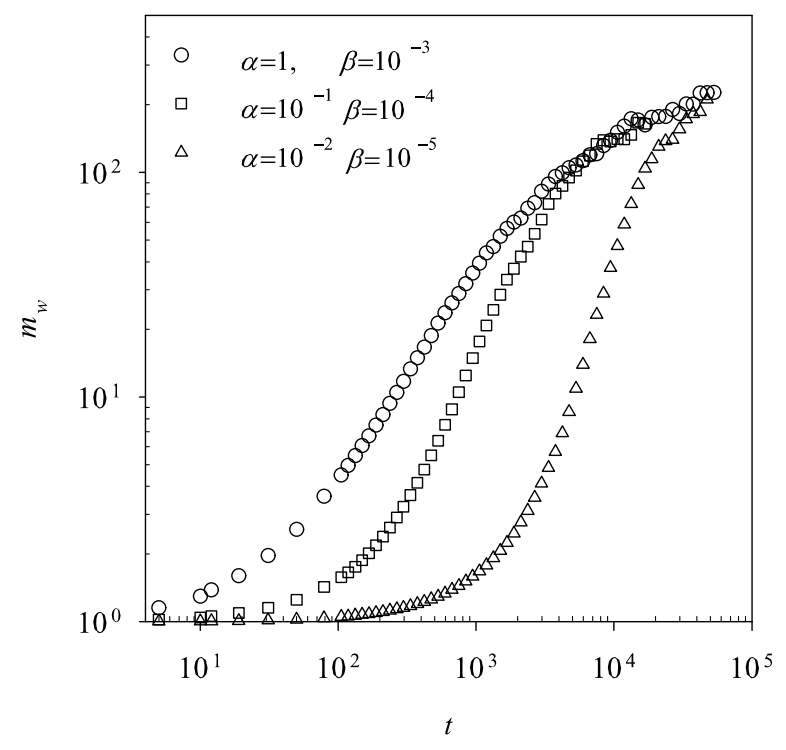

FIG. 3: Comparison of the evolution of $m_{w}$ of different values of $\alpha$ for the same value of $t_{e}=1430$.

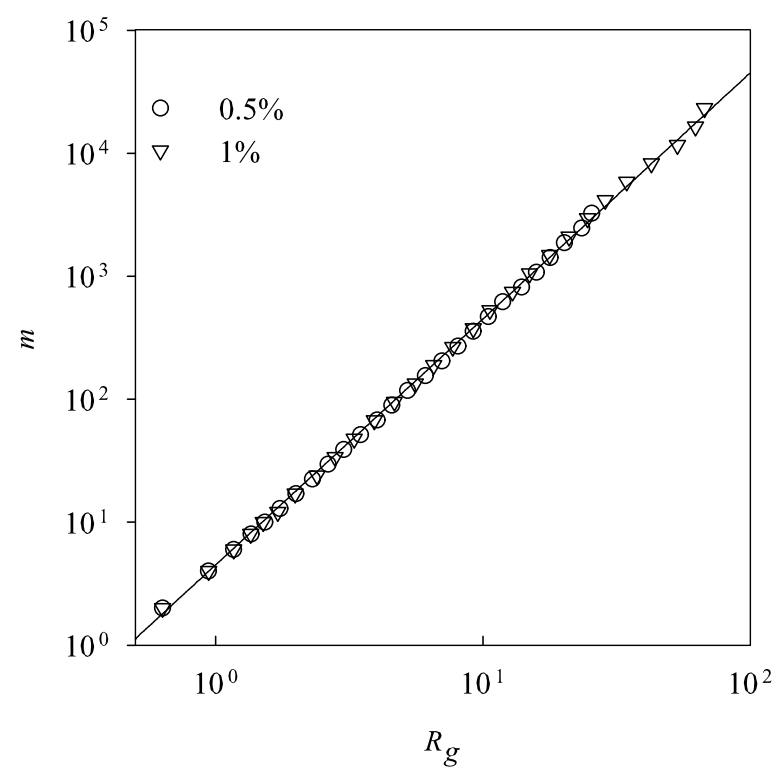

FIG. 4: Dependence of the aggregation number on the radius of gyration for $\phi=0.5 \%$ and $1 \%$. The straight line represents a linear least square fit and has a slope of 2.0.

means that the clusters have a self similar structure with $d_{f}=2.0$. The same value was found by Odriozola et al. from simulations of reversible DLCA. A fractal dimension of 2 is expected if each configuration has equal probability [36-38], which is the case for reversible aggregation where the clusters restructure rapidly compared to their diffusion rate. Fig.5 shows that the size distributions at

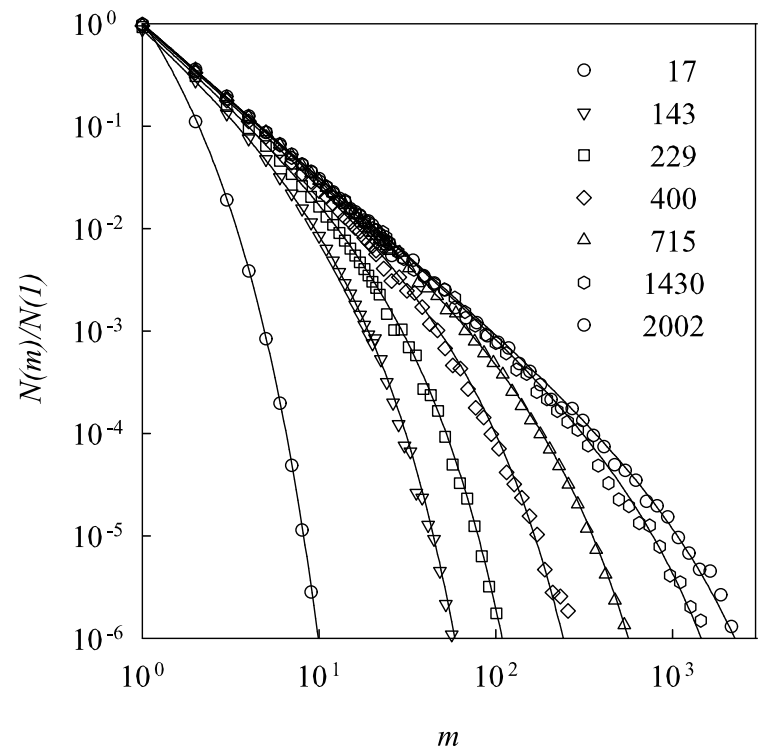

FIG. 5: Cluster size distribution for $\phi=0.5 \%$ at different values of $t_{e}$ indicated in the figure. The solid lines represent the fits to Eq.4.

different values of $t_{e}$, can be well described by a power law decay with an exponential cut-off at a characteristic aggregation number $m^{*} \propto m_{w}$ :

$$
N(m)=\kappa \cdot m^{-\tau} \cdot \exp \left(-m / m^{*}\right)
$$

where $N(m)$ is the fraction of clusters with aggregation number $m$ and $\tau=1.5 . \kappa$ is related to $m^{*}$ by the condition of mass conservation $\sum m \cdot N(m)=1$, which gives $\kappa=\left(m^{*(2-\tau)} \cdot \Gamma(2-\tau)\right)^{-1}$ for $m^{*} \gg 1$. Utilizing Eq. 4 in Eqs 2 and 3 it follows that:

$$
\begin{gathered}
m_{w}=\frac{1}{2 \pi \cdot \kappa^{2}} \quad m_{w} \gg 1 \\
m_{n}=\frac{1}{2 \kappa} \quad m_{n} \gg 1
\end{gathered}
$$

If aggregation and fragmentation of the clusters does not depend on the position of the clusters, the time dependence of $N(m)$ can be written as [15]

$$
\begin{array}{r}
\frac{d N(m)}{d t}=\frac{1}{2} \sum_{i+j=m}[K(i, j) N(i) N(j)-F(i, j) N(m)] \\
-\sum_{j}[K(m, j) N(m) N(j)-F(m, j) N(j+m)]
\end{array}
$$

where $K(i, j)$ is the mean rate with which a cluster with size $i$ binds to a cluster with size $j$, and $F(i, j)$ is the mean rate with which a cluster breaks up into two clusters with 
size $i$ and $j$. In equilibrium the number of clusters of a given size stays constant and the condition of detailed balance gives:

$$
K(i, j) \cdot N(i) \cdot N(j)=F(i, j) \cdot N(m)
$$

If the collisions between the particles are uncorrelated the collision rate can be described by the Brownian kernel [39]:

$$
K(i, j)=4 \pi \cdot\left(R_{c o l, i}+R_{c o l, j}\right) \cdot\left(D_{i}+D_{j}\right)
$$

where $R_{c o l, i} \propto i^{1 / d_{f}}$ is the collision radius and $D_{i} \propto$ $i^{-1 / d_{f}}$ is the diffusion coefficient of clusters with size $i$. At each collision the clusters form a bond with average life time: $s^{2} \cdot \alpha / \beta$, see appendix 1 .

For $m_{w} \ll 1$ the average molar aggregation number is determined essentially by the monomer-dimer equilibrium. The concentration of encounters between two spheres, $C_{e}$, is a function of the rate of formation and the rate of break-up of an encounter: $C_{e}=$ $C \cdot K(1,1) / F(1,1)$, considering that the number concentration of spheres $(C=6 \phi / \pi)$ is much larger than $C_{e}$. $K(1,1)$ is the collision rate of uncorrelated spheres, which is $4 \pi / 3$ in our simulations, because the spheres have radius 0.5 and diffusion coefficient $1 / 6 . F(1,1)$ is the rate at which the two monomers become decorrelated, which is equal to the inverse of the life time of an encounter $\left(t_{e}\right)$. The fraction of dimers $(N(2))$ is proportional to the number of encounters: $N(2) \propto \phi \cdot t_{e}$. It follows that:

$$
m_{n}-1=\frac{\left(m_{w}-1\right)}{2} \propto \phi \cdot t_{e} \quad m_{w}-1 \ll 1
$$

The dependence of $m_{n}-1$ and $m_{w}-1$ on $\phi \cdot t_{e}$ is shown in Fig. 6 for $0.5 \%$ and $1 \%$ and is well described by Eq.10 for $m_{w}-1 \ll 1$ using a proportionality factor of 2.2 .

Remarkably, $m_{n}$ has the same dependence on $\phi \cdot t_{e}$ also for large values, while $m_{w}$ has a steeper dependence. The same dependence of $m_{n}$ on $\phi \cdot t_{e}$ is only obtained for $m_{n} \gg 1$ if $\kappa$ in Eq. 6 is equal to $0.23\left(\phi \cdot t_{e}\right)^{-1}$, which means:

$$
m_{n}=2.2 \phi \cdot t_{e} \text { and } m_{w}=3.0\left(\phi \cdot t_{e}\right)^{2} \quad m_{w} \gg 1
$$

The dashed line in Fig.6 shows that the simulation results for $m_{w}$ approach the power law dependence asymptotically. Unfortunately, we are at the present stage not able to use box sizes with $L>200$ that are necessary to simulate equilibrium states with even larger $m_{w}$.

For $m_{w}>>1$ the cluster size distribution in equilibrium is given by Eq.4 if $K(i, j)$ and $F(i, j)$ are homogeneous $\left(K(a i, a j)=a^{\lambda} \cdot K(i, j)\right.$ and $\left.F(a i, a j)=a^{\theta} \cdot F(i, j)\right)$ and if the fragmentation kernel may be written as [40]:

$$
F(i, j)=\kappa \cdot K(i, j) \cdot\left(i^{-1}+j^{-1}\right)^{\tau}
$$

The implication is that $\tau=\lambda-\theta$. For the Brownian collision kernel $\lambda=0$ so that $\theta=-\tau$.

The clusters have a self similar structure and contain no loops. This would suggest that the number of bonds

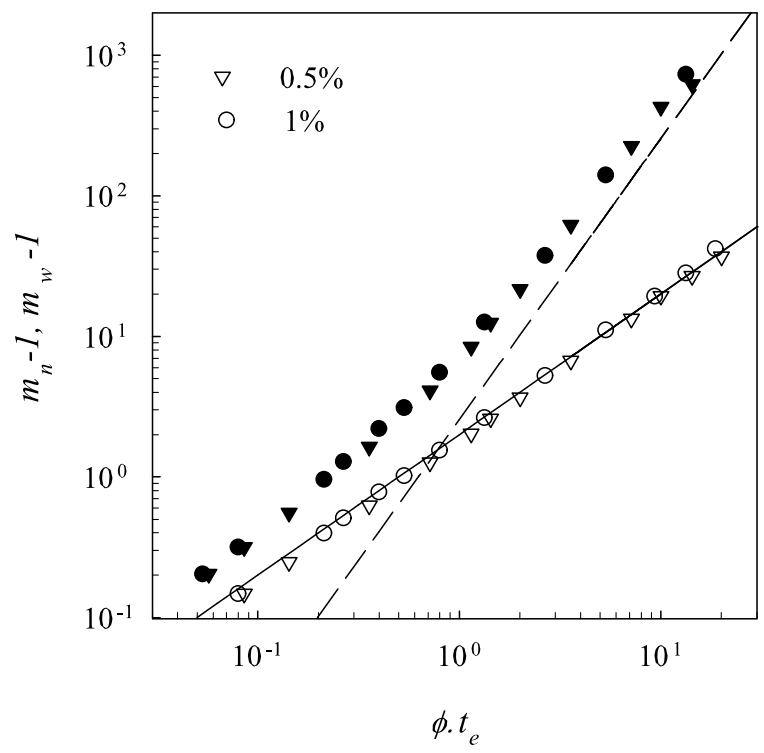

FIG. 6: Dependence of the weight (open symbols) and the number averaged (closed symbols) aggregation number as a function of $\phi \cdot t_{e}$ for $\phi=0.5 \%$ and $1 \%$. The solid line represents $m_{n}=2.2 \phi \cdot t_{e}$ and the dashed line $m_{w}=3.0\left(\phi \cdot t_{e}\right)^{2}$.

$\left(e_{i j}\right)$ that connect a cluster with size $i$ to a cluster with size $j=m-i$ is proportional to $m \cdot i^{-1}$ for $m \gg 1$ and choosing $i<j$. Odriozola et al. [34] found using computer simulations : $e_{i, j}=0.439 m \cdot\left(i^{-1}+j^{-1}\right) \cdot(i \cdot j)^{-0.136}$. The term $(i \cdot j)^{-0.136}$ is not expected for self similar clusters without loops if each bond has the same probability to break. If each bond breaking leads to decorrelation of the fragments then the fragmentation kernel would be: $F(i, j) \propto \beta /\left(s^{2} \cdot \alpha\right) \cdot i^{-1}$.

However, the fragments become decorrelated only if they diffuse apart without colliding again. In our simulations monomers move at each step, but the probability of relative movement for two larger clusters is equal to $\left(D_{i}+D_{j}\right)$. Even if the fragments move apart they have a high probability to collide again. If we assume that the number of correlated collisions between the two fragments is equal to that found for two monomers $\left(N_{c o l}(i, j)=N_{c o l}(1,1)\right)$, then the average total bond life time between the fragments is proportional to $t_{e} /\left(D_{i}+\right.$ $\left.D_{j}\right)$. One may thus attempt to account for the correlation between collisions by writing the fragmentation kernel as:

$$
F(i, j) \propto \frac{\left(D_{i}+D_{j}\right)}{t_{e} \cdot i}
$$

The homogeneity exponent of this kernel is $\theta=-(1+$ $\left.d_{f}^{-1}\right)=-1.5$, which is consistent with the simulation 
results.

Odriozola et al. [34] have deduced the number of correlated collisions explicitly as a function of $i$ and $j$ from computer simulations of RLCA. They obtained: $N_{c o l}(i, j)=N_{c o l}(1,1) \cdot(i \cdot j)^{0.35}$, but it is unclear why this result obtained on irreversibly bonded clusters is relevant for transient clusters with a very short life time compared to their diffusion rate. Nevertheless, they found that the fragmentation kernel obtained in this way could well describe the evolution of $m_{w}$ in reversible DLCA. We note that the homogeneity exponent of their fragmentation kernel is very close to that of Eq.13: $\theta=-1.49$. However, neither the fragmentation kernel proposed by Odriozola nor Eq.13 is consistent with the condition of detailed balance (Eq.8) if the Brownian kernel is used to describe the collision rate. One may even wonder whether using a mean field theory (Eq.7) is justified for the case of reversible aggregation where collisions are highly correlated. In addition, as we have discussed above, the fragments can only move a very small distance before they break again.

\section{PERCOLATION}

Flocculation describes the aggregation process of highly diluted systems when the clusters are on average far apart although the collisions may still be highly correlated, as we have seen in the previous section. However, for any given volume fraction the average distance between the clusters decreases with increasing $t_{e}$, because the size of the clusters increases and their density decreases. The cumulated volume fraction of the clusters can be calculated as:

$$
V_{\text {cum }}=N \cdot \sum_{m=1}^{\infty} \frac{N(m)}{L^{3}} \cdot \frac{4 \pi}{3} \cdot R_{g}^{3}(m)
$$

In the flocculation regime Eq.14 becomes:

$$
V_{\text {cum }} \propto \phi \cdot \kappa \int_{1}^{\infty} m^{\frac{3}{d_{f}}-\tau} \cdot \exp \left(-m / m^{*}\right) d m
$$

where we have used Eq.4. It follows that $V_{\text {cum }} \propto \phi \cdot \sqrt{m_{w}}$ for $m_{w} \gg 1$ because $m_{w} \propto m^{*}$. At any volume fraction a transition from flocculation to percolation occurs when the clusters start to overlap, i.e. when $V_{\text {cum }}$ is of order unity. As a consequence of this transition the structure and the size distribution of clusters change for clusters with $m$ larger than a characteristic value $m_{c} \propto \phi^{-2}$.

The change in the fractal structure between flocculated clusters and percolated clusters is seen in Fig.7. In order to demonstrate the universality of the cross-over at $m_{c}$ we have plotted $m / R^{2}$ as a function of $m / m_{c}$. This representation also allows for an accurate determination of the fractal dimensions in the limiting regimes of flocculation $\left(m \ll m_{c}\right)$ and percolation $\left(m \gg m_{c}\right)$ $\left(m / R^{2}=\left(m / m_{c}\right)^{1-2 / d_{f}}\right)$. In the flocculation regime $d_{f}=2.0$, while in the percolation regime $d_{f}=2.7$, which

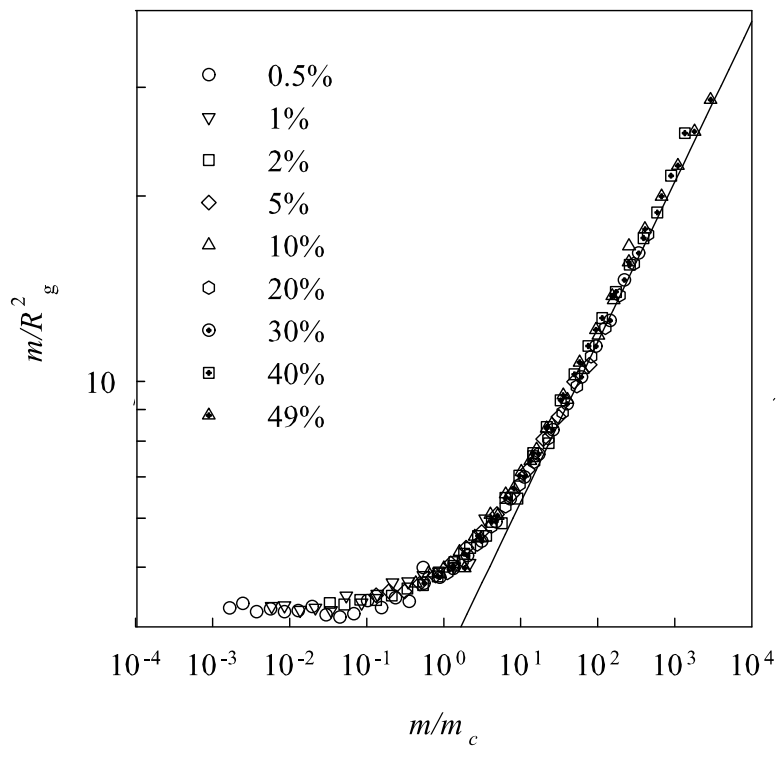

FIG. 7: Dependence of $m / R_{g}^{2}$ as a function of $m / m_{c}$ for different volume fractions indicated in the figure. The solid line has a slope of 0.37 .

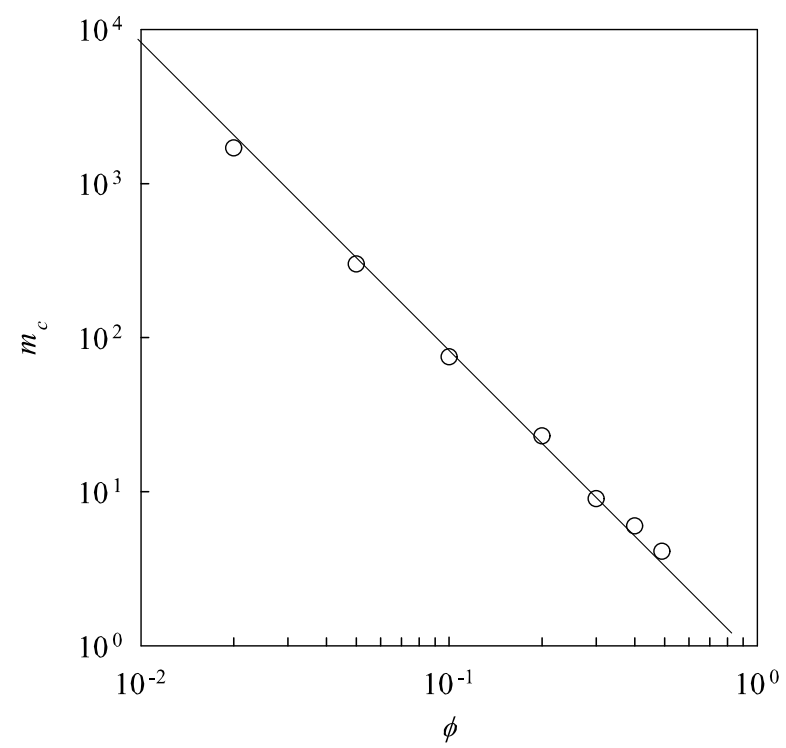

FIG. 8: Dependence of $m_{c}$ on the volume fraction. The solid line has a slope -2 .

is slightly above the value found for random percolation $[30,41]$ on lattices and irreversible aggregation $\left(d_{f}=2.5\right)$ $[13,14,28]$. The dependence of $m_{c}$ on the volume fraction is compatible with $m_{c} \propto \phi^{-2}$, see Fig.8.

The number of percolating clusters with size $m$ decreases with $m$ following a power law as in the flocculation regime, but the exponent $\tau$ increases from the floccu- 


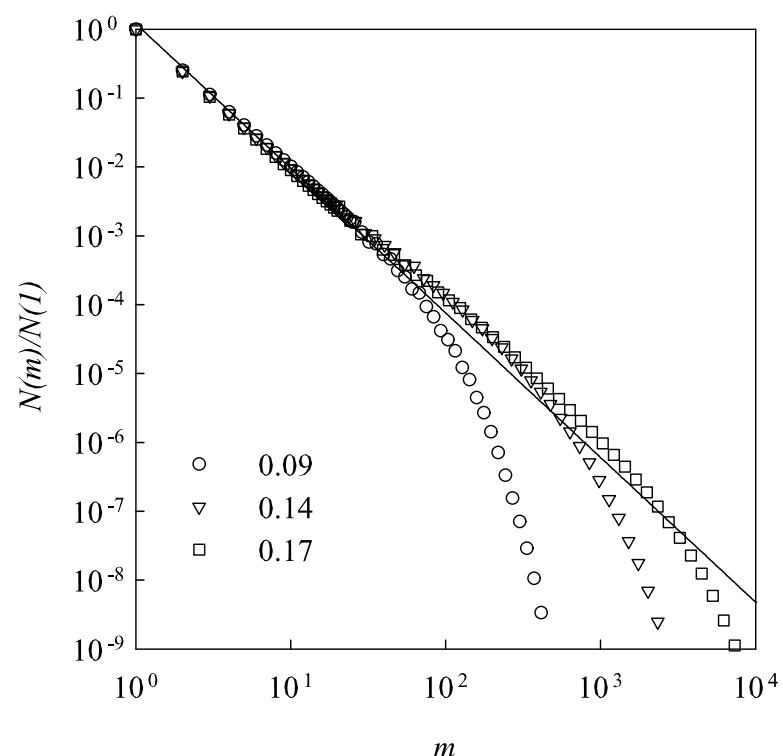

FIG. 9: Cluster size distributions at different values of $t_{e}$ indicated in the figure for $\phi=49 \%$. The solid lines has a slope of -2.1 .

lation value 1.5 for $m \ll m_{c}$ to a larger value for $m \gg m_{c}$. In addition, the cut-off function at $m=m^{*}$ is no longer a simple exponential. For percolating clusters $\tau$ and $d_{f}$ are related by the so-called hyper scaling law: $d_{f}=d /(\tau-1)$, with $d$ the spatial dimension. Simulations of site percolation on a cubic lattice gave $\tau=2.2$ consistent with the hyper scaling law $[30,41]$. The percolation regime is best observed for simulations at high volume fractions where $m_{c}$ is small. Fig.9 shows cluster size distributions at different $t_{e}$ for $\phi=0.49$ where $m_{c}=3.3$. For comparison a line representing $\tau=2.1$ is shown, which is the value predicted by the hyper scaling law using $d_{f}=2.7$. The power law decrease of $N(m)$ is observed only in a relatively narrow region, because finite size effects limit the exploration of very large clusters. At intermediate concentrations a cross-over between $\tau=1.5$ and $\tau=2.1$ occurs at $m \approx m_{c}$.

Assuming that $\tau=2.1$ we can obtain the cut-off function of the size distribution by plotting $m^{2.1} \cdot N(m) / N$ as a function of $m / m_{z}$ for $m \gg m_{c}$, where $m_{z} \approx m^{*}$ is the z-average aggregation number. Fig.10 shows the cutoff functions of size distributions close to the percolation threshold for a few high volume fractions. For static percolation on a cubic lattice a Gaussian cut-off function was found $[30,41]$. In the present case the cut-off function has again the form of a Gaussian, but not with the same numerical constants. In addition, the shape of the cutoff function depends slightly on the volume fraction. It thus appears that the structure and the size distribution of percolating clusters obtained from reversible off-lattice simulations is slightly different from those obtained from random percolation on a cubic lattice. The fractal dimension of percolating clusters obtained for on- and off-

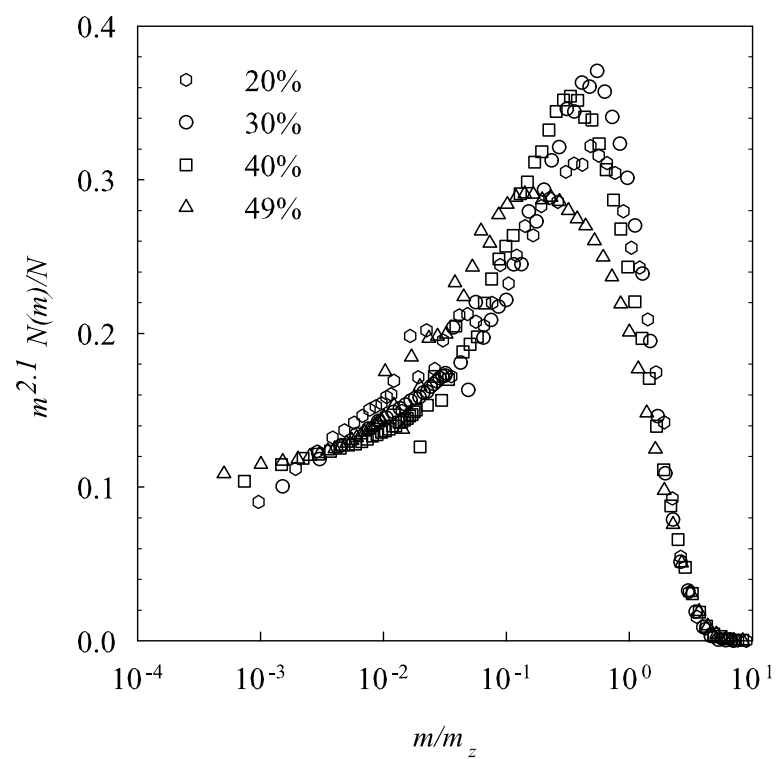

FIG. 10: Cut-off functions of size distributions close to the gel point at different volume fractions indicated in the figure.

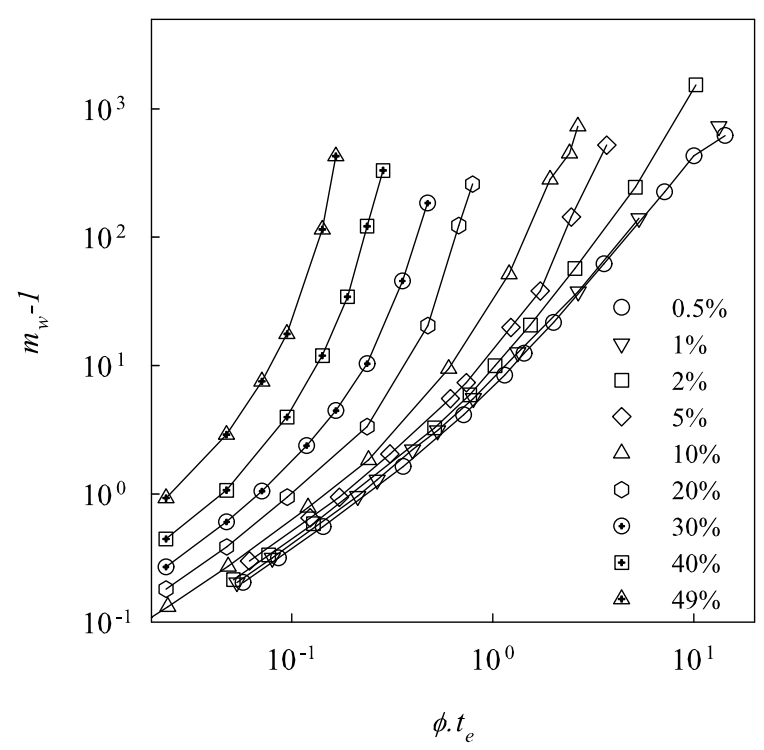

FIG. 11: Dependence of the weight average aggregation number on $\phi \cdot t_{e}$ for different volume fractions indicated in the figure.

lattice simulations of irreversible DLCA is the same as found for random percolation. Unfortunately, these simulation results were not sufficiently precise to distinguish between $\tau=2.2$ and $\tau=2.1$.

For irreversible aggregation the transition from flocculation to percolation causes an acceleration of the growth rate $[14,28]$. Fig.11 shows that also for reversible aggre- 


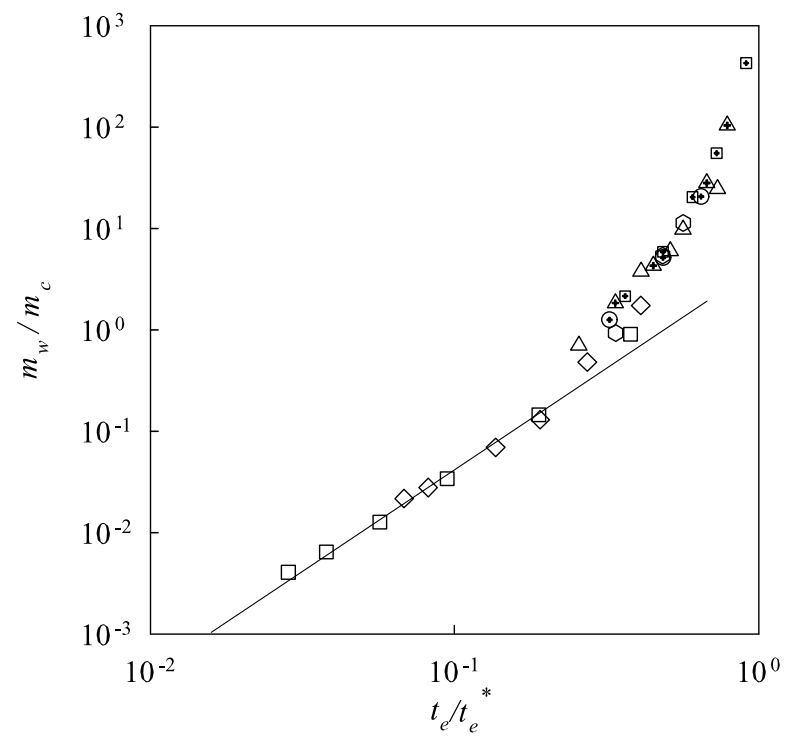

FIG. 12: Representation of the data shown in Fig.11 for $\phi \geq$ $2 \%$ and $m_{w}>10$ in terms of the reduced parameters $m_{w} / m_{c}$ and $t_{e} / t_{e}^{*}$. The solid line has a slope of 2 .

gation the transition causes an increase of the dependence of $m_{w}$ on $t_{e}$ for $m_{w}>m_{c}$. At each volume fraction $m_{w}$ diverges at a critical value of $t_{e}=t_{e}^{*}$ where the system percolates, while $m_{n}$ remains finite. If the flocculationpercolation transition is universal then $m_{w} / m_{c}$ should be a universal function of $t_{e} / t_{e}^{*}$ for $m_{w} \gg 1$, as was reported earlier for lattice and off-lattice simulations of irreversible aggregation $[14,28]$. Fig.12 shows that a universal transition is also found for reversible aggregation.

The dependence of $t_{e}^{*}$ on $\phi$ is shown in Fig.13. In the flocculation regime, i.e $m_{w} / m_{c} \propto\left(t_{e} / t_{e}^{*}\right)^{2}$, which implies that $t_{e} \propto \phi^{-2}$, because $m_{w} \propto \phi^{2}$ and $m_{c} \propto \phi^{-2}$. A line with slope -2 is drawn for comparison in Fig.13. Of course, this behaviour is only expected if the concentration is low enough so that the initial growth occurs in the flocculation regime.

In this work reversible aggregation of spheres was studied for the case that the bonds are rigid and formed only at contact. As mentioned in the introduction this implies that only binary collisions are possible and no densification can occur. We verified that in all cases each sphere was bound to on average $2 \cdot\left(1-1 / m_{n}\right)$ neighbors. Therefore phase separation does not occur at any bond-breaking probability. Elsewhere we will show that if bond formation may occur not only at contact, but also if the particles are within a finite attraction range then a square well potential is mimicked and phase separation is observed if the attraction is sufficiently strong.

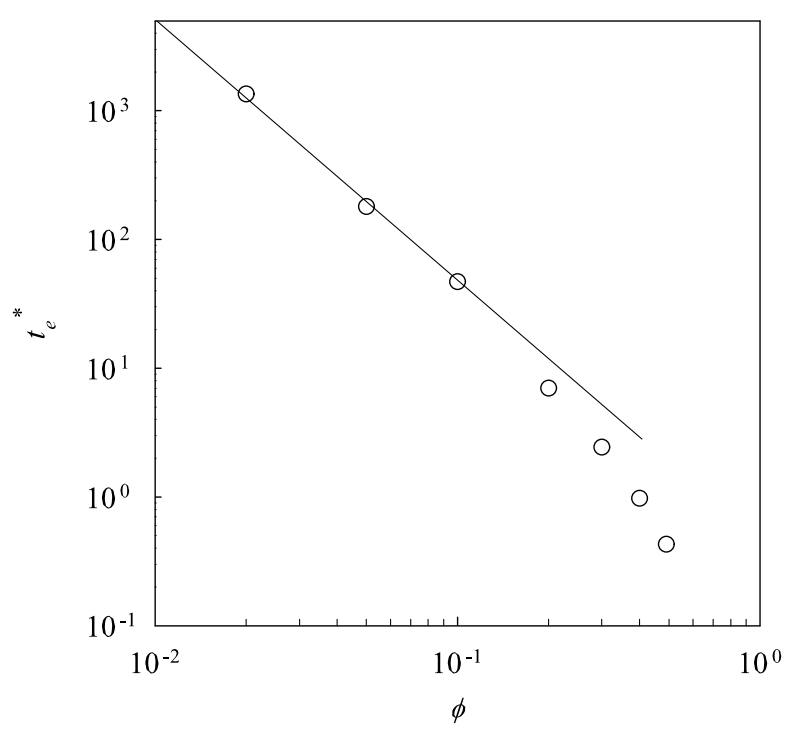

FIG. 13: Dependence of the critical life time on the volume fraction. The solid line has a slope of -2 .

\section{CONCLUSION}

The equilibrium properties of clusters formed by reversible aggregation are determined by the average life time of an encounter between two particles $\left(t_{e}\right)$. The latter is not only a function of the bond formation $(\alpha)$ and break-up $(\beta)$ probabilities, but also of the elementary step size $(s)$ of the Brownian motion of the particles. At the same value of $t_{e}$ the equilibrium state is the same both in the diffusion controlled limit $(\alpha=1)$ and in the reaction controlled limit $(\alpha \ll 1)$. If the clusters are on average far apart, i.e. in the flocculation regime, then $d_{f}=2.0$ while the size distribution has a power law decay with exponent -1.5 and an exponential cutoff. A transition to the percolation regime occurs with increasing $t_{e}$ when the clusters start to overlap. At each volume fraction a transient gel is formed above a critical life time $t_{e}^{*}$. $t_{e}^{*}$ decreases with increasing volume fraction and is proportional to $\phi^{-2}$ for small $\phi$. Close to the percolation threshold the clusters have a fractal dimension $d_{f}=2.7$ while the size distribution has power law decay with exponent $\tau=2.1$ and a Gaussian cut-off. The transition between flocculation and percolation occurs at a characteristic weight average aggregation number that decreases with increasing volume fraction: $m_{c} \propto \phi^{-2}$.

\section{APPENDIX 1}

The probability that two monomers that collide form a bond is $\alpha$. After the bond is formed it is broken with probability $\beta$ at each simulation step.

The probability that the bond lifetime is $0,1,2$ or $i$ 
simulation steps is: $P_{0}=1-\alpha, P_{1}=\alpha \cdot \beta, P_{2}=\alpha$. $\beta \cdot(1-\beta)$ or $P_{i}=\alpha \cdot \beta \cdot(1-\beta)^{i-1}$ respectively. The average bond life time per collision in simulation steps is $\sum_{i} i \cdot P_{i}$, which gives:

The average physical life time $\left(t_{e}\right)$ is thus:

$$
t_{e}=N_{c o l} \cdot s^{2} \cdot \frac{\alpha}{\beta}
$$

\section{APPENDIX 2}

The probability that at least one of the bonds of a cluster of size $m$ breaks in a single simulation step is:

$$
1-(1-\beta)^{m-1}
$$

The probability that at least one bond breaks after $i$ simulation steps is:

$$
P(i)=\left[1-(1-\beta)^{m-1}\right] \cdot(1-\beta)^{i \cdot(m-1)}
$$

The average number of simulation steps during which they remain intact is:

$$
\sum_{i=1}^{\infty} i \cdot P(i)=\frac{(1-\beta)^{m-1}}{1-(1-\beta)^{m-1}}
$$

$$
\alpha \cdot \beta \cdot \sum_{i=1}^{\infty} i \cdot(1-\beta)^{i-1}=\frac{\alpha}{\beta}
$$

The average life time of a cluster $\left(t_{l}\right)$ is thus:

$$
t_{l}=s^{2} \cdot \frac{(1-\beta)^{m-1}}{1-(1-\beta)^{m-1}}
$$

Large clusters with $m \gg 1$ can only be formed for $m \cdot \beta \ll$ 1 which gives:

$$
t_{l}=\frac{s^{2}}{m \cdot \beta}
$$

The average distance a cluster diffuses during that time is:

$$
<r^{2}>=\frac{t_{l}}{m^{1 / d_{f}}}
$$

\section{Acknowledgment}

This work has been supported in part by a grant from the Marie Curie Program of the European Union numbered MRTN-CT-2003-504712.

Technol. 15, 129 (1999).

[1] M. Carpineti, F. Ferri, M. Giglio, E. Paganini, and U. Perini, Phys. Rev. A 42, 7347 (1990).

[2] T. Nicolai and S. Cocard, Eur. Phys. J. E 5, 221 (2001).

[3] C. G. de Kruif, M. A. M. Hoffmann, M. E. van Marle, P. J. J. M. van Mil, S. P. F. M. Roefs, M. Verheul, and N. Zoon, Faraday Discuss. 101, 185 (1995).

[4] S. H. Chen, J. Rouch, F. Sciortino, and P. Tartaglia, J. Phys.: Condens. Matter 6, 10855 (1994).

[5] P. Meakin, Phys. Rev. Lett. 51, 1119 (1983).

[6] M. Kolb, R. Botet, and R. Jullien, Phys. Rev. Lett. 51, 1123 (1983).

[7] A. Hasmy, M. Foret, J. Pelous, and R. Jullien, Phys. Rev. B 48, 9345 (1993).

[8] M. Lattuada, P. Sandkuhler, H. Wu, J. Sefcik, and M. Morbidelli, Adv. Colloid Interface Sci. 103, (2003).

[9] P. Meakin, Phys. Rev. A 38, 4799 (1988).

[10] G. Odriozola, A. Moncho-Jorda, A. Schmitt, J. CallejasFernandez, R. Martinez-Garcia, and R. Hidalgo-Alvarez, Europhys. Lett. 53, 797 (2001).

[11] M. Kolb and H. J. Herrmann, J. Phys. A: Math. Gen. 18, L435 (1985).

[12] A. Hasmy and R. Jullien, J. Non-Cryst. Solids 186, 342 (1995).

[13] J. C. Gimel, D. Durand, and T. Nicolai, Phys. Rev. B 51, 11348 (1995).

[14] J. C. Gimel, T. Nicolai, andD. Durand, J. Sol-Gel Sci.
[15] F. Family, P. Meakin, and J. M. Deutch, Phys. Rev. Lett. 57, 727 (1986).

[16] M. Kolb, J. Phys. A: Math. Gen. 19, L263 (1986).

[17] W. Y. Shih, I. A. Aksay, and R. Kikuchi, Phys. Rev. A 36, 5015 (1987).

[18] M. D. Haw, M. Sievwright, W. C. K. Poon, and P. N. Pusey, Adv. Colloid Interface Sci. 62, 1 (1995).

[19] J. C. Gimel, T. Nicolai, and D. Durand, Eur. Phys. J. E 5, 415 (2001).

[20] G. Odriozola, A. Schmitt, A. Moncho-Jorda, J. CallejasFernandez, R. Martinez-Garcia, R. Leone, and R. Hidalgo-Alvarez, Phys. Rev. E 65, 031405 (2002).

[21] M. T. A. Bos and J. H. J. van Opheusden, Phys. Rev. E 53, 5044 (1996).

[22] K. G. Soga, J. R. Melrose, and R. C. Ball, J. Chem. Phys. 110, 2280 (1999).

[23] J. F. M. Lodge and D. M. Heyes, Phys. Chem. Chem. Phys. 1, 2119 (1999).

[24] A. Coniglio, L. de Arcangelis, E. del Gado, A. Fierro, and N. Sator, J. Phys.: Condens. Matter 16, S4831 (2004).

[25] S. A. Safran, I. Webman, and G. S. Grest, Phys. Rev. A 32, 506 (1985).

[26] J.-M. Jin, K. Parbhakar, L. H. Dao, and K. H. Lee, Phys. Rev. E 54, 997 (1996).

[27] T. Terao and T. Nakayama, Phys. Rev. E 58, 3490 
(1998)

[28] M. Rottereau, J. C. Gimel, T. Nicolai, and D. Durand, Eur. Phys. J. E 15, 133 (2004).

[29] M. Rottereau, J. C. Gimel, T. Nicolai, and D. Durand, Eur. Phys. J. E 15, 141 (2004).

[30] D. Stauffer and A. Aharony, Introduction to percolation theory, second edition ed. (Taylor \& Francis, London, 1992).

[31] M. G. Noro and D. Frenkel, J. Chem. Phys. 113, 2941 (2000).

[32] M. E. Cates, M. Fuchs, K. Kroy, W. C. K. Poon, and A. M. Puertas, J. Phys.: Condens. Matter 16, S4861 (2004).

[33] J. C. Gimel, T. Nicolai, and D. Durand, Phys. Rev. E 66, 061405 (2002).

[34] G. Odriozola, A. Schmitt, A. Moncho-Jorda, J. Callejas-
Fernandez, R. Martinez-Garcia, R. Leone, and R. Hidalgo-Alvarez, Phys. Rev. E 65, 031405 (2002).

[35] M. Rottereau, J. C. Gimel, T. Nicolai and D. Durand, Eur. Phys. J. E. 18, 15 (2005).

[36] R. Wessel and R. C. Ball, Phys. Rev. A 45, R2177 (1992).

[37] R. Wessel and R. C. Ball, J. Phys. A: Math. Gen. 26, L159 (1993).

[38] G. Parisi and N. Sourlas, Phys. Rev. Lett. 46, 871 (1981).

[39] S. Chandrasekhar, Rev. Mod. Phys. 15, 1 (1943).

[40] M. H. Ernst and P. G. J. van Dongen, Phys. Rev. A 36, 435 (1987).

[41] J. C. Gimel, T. Nicolai, and D. Durand, J. Phys. A: Math. Gen. 33, 7687 (2000). 